The minor phyla still get scant attention and the chapter on phylogeny is too brief to say anything much of worth, but that on fossils is greatly improved by the many photographs. The chapter which considers how and where zoologists carry out their research and communicate their findings will give the novice an idea of how the information given elsewhere in the book came to light. There is a short section on classification, and a list of books and journals.

The same four authors have produced Living Invertebrates, a more comprehensive work designed for a more advanced readership. Many of the drawings by Mildred Waltrip appear in both books, and helpful photographs abound (with 35 colour pages illustrating the chapter on pigments and colouration). A book list is included, but again there are no references to original papers in journals.

Unfortunately, the chatty and rather naive style of writing, which works well at the level of Animals Without Backbones, is here too. In the context of this larger and more serious-minded book one can easily find it irritating. At the level at which this book is pitched, it is not a virtue to avoid technical terms wherever possible - such words have a precise meaning and therefore a very real value. Students are quite capable of mastering a remarkable number of them, particularly if they learn their etymology, and it is impoverishing the language to shun them. So the book falls between two stools, with neither the freshness and simplicity which makes Animals Without Backbones so appealing to the newcomer to invertebrates nor the depth of treatment and scholarship shown by Barnes's Invertebrate Zoology.

Now in its fifth edition, Invertebrate Zoology has always been a very good book for the serious student, for it contains a great deal of information, is well illustrated and has plenty of references. Each edition was getting ever more weighty, however, and I gently took the author to task in these columns when considering the fourth edition. Apparently it was generally felt to be too large and a reduction of about 170 pages has been made. New material is included, however, and there are two distinct improvements: an expanded glossary combined with the index, and 'boxed essays'. These are short treatments of topics which recur in various invertebrate groups (such as trochophore larvae, eyes, nephridia, bioluminescence) and they provide useful overviews and comparisons.

So Barnes is better than ever, though it's still the case that only 3 per cent of the space is given to the insects. But then, nobody's perfect.

Alan Brafield is a Senior Lecturer in the Department of Biology, King's College London ment of Biology, Kill Road, London W8 $7 A H, U K$

\section{Organic evolution}

\section{John Mann}

Organic Chemistry. By K. Peter C. Vollhardt. W.H. Freeman: 1987. Pp. 1,275 plus answers to exercises and index. $H b k$ $\$ 49.95, £ 49.95$; pbk \$33.95, £18.95. (Study guide and solutions manual, pbk \$20.95, f16.95).

Organic Chemistry. By Francis A. Carey. McGraw-Hill: 1987. Pp. 1,219. \$45.95, $£ 43.95$.

Basic Organic Chemistry: A Mechanistic Approach, 2nd Edn. By J. M. Tedder and A. Nechvatal. Wiley: 1987. Pp. 303. Hbk $£ 33, \$ 69 ; p b k £ 9.95, \$ 19.95$.

The Art of Problem Solving in Organic Chemistry. By Miguel E. Alonso. Wiley: 1987. Pp.324. £25.95, \$29.95.

THE standard textbook of organic chemistry has been evolving continuously for the past 30 years. Like the movies, it began in black and white, and has progressed stage by stage until now it is multicoloured. In the 1960 s we had the arrival or new editions of the excellent books by the Fiesers (Advanced Organic Chemistry) and Finar (Organic Chemistry), and the somewhat dull and factual Organic Chemistry by Morrison and Boyd

The latter two books, currently in their sixth editions, are still with us, and although Morrison and Boyd now includes colour, Finar has retained its classical monochrome appearance. One feature that raised the Fiesers' book (and to a lesser extent Finar's) above all others, then and since, was that it read like a novel. All the essential facts were reported, but interspersed with short biographies of famous chemists, and interesting pieces on the industrial relevance of the topics discussed. The book was brim full with the excitement of organic chemistry.

In the intervening years much of this excitement has gone from textbooks, to be replaced by lists of reactions and masses of problems; and all the 'advanced' topics are confined to boxes or appendices where they cannot disturb the learning process. The market for these books is nonetheless enormous, and there is a seemingly annual competition to produce the new text with the most novel features.

In 1987, Peter C. Vollhardt's Organic Chemistry wins hands down. It employs four colours to simplify reaction mechanisms, to identify signals in spectra and to clarify functional-group interconversions; and the depictions of three-dimensional structures (and of orbitals) are not only clear but very appealing. The content is much the same as that of other recent textbooks, though there is a strong bias towards organic synthesis, and the applications of organic chemistry to the biological sciences and to industry are stressed. One nice touch is that all of the reactions reported were checked in the literature, or were run in the laboratory to confirm their authenticity. There is also a good chapter on heterocyclic chemistry.

Overall, the book is visually appealing, up-to-date and, although it does not read like a novel, it is informative and easy to follow. It is hard to imagine next year's novelty, though three-dimensional 'popup' structures might be one possibility!

It is rather unfortunate that Francis A. Carey's book has to be reviewed at the same time as Vollhardt. It, too, is eminently suitable for undergraduate classes, has plenty of organic synthesis and other modern features; but only three colours are employed, and they are not used as imaginatively as in Vollhardt's book. Carey has some advantages over Vollhardt, in that classes of compounds (such as organometallic reagents and isoprenoids) are dealt with all together, and are not dispersed through several chapters. But, overall, the consumer is likely to choose Vollhardt for its sheer good looks.

British textbooks of organic chemistry are a comparative rarity, and so the second edition of Tedder and Nechvatal's Basic Organic Chemistry is to be welcomed. The first edition appeared 20 years ago, and one of the main developments since then has been the increasing application of spectroscopic techniques in organic chemistry. Most chapters of this new edition begin with a summary of the main spectral features (principally NMR and infrared) of the functional group under discussion, but otherwise the authors concentrate on basic mechanisms.

The text is surprisingly comprehensive for its 300 pages, and the authors even manage to include brief accounts of natural products, carbohydrates, polymers and molecular orbital theory. But this book will probably be mainly of use to first-year chemistry undergraduates or to biologists requiring a grounding in organic chemistry. One annoying feature is the poor quality of the printing, and in visual appeal the volume compares very unfavourably with the two previous books.

Miguel E. Alonso's The Art of Problem Solving in Organic Chemistry is very different from the other books reviewed here. It contains 56 problems in synthesis, with worked answers, but these are probably too difficult for most undergraduates. They will, however, be ideal for the postgraduate. The illustrations range in quality from good to confusing, but the answers are usually very helpful. This book will be an essential purchase for all $\mathrm{PhD}$ supervisors, allowing them to set problems without having to comb the research literature - and thus freeing them to concentrate on raising money.

John Mann is a Lecturer in the Department of Chemistry, University of Reading, Whiteknights, Reading RG6 $2 A D, U K$. 